\title{
Author Index Vol. 62, 1995
}

Abe,M. 269 Abe,S. 64,84, 110,317 Adelmann-Grill, B.C. 209 Akino,T. 64 Amir, E. 16 An,D. 307 Andersson, S.E. 34 Antypas, G. 160 Arapantoni-Dadioti, P. 160

Baglioni, S. 16 Balcells,E. 353 Baldi,S. 201 Baydur,A. 104 Behera,D. 10,263 Behr,J. 209

Beinert,T. 209 Benlloch, E. 4 Bergandi,D. 228 Bernard, S. 10,307 Biasin,C. 95 Blanco-

Aparicio, M. 53 Bofill, J.M. 353 Bollo,E. 258 Bouros,D. 280,286,336 Boyer, S. 130 Bozzoni,

M. 331 Brussino, L. 228 Bucca,C. 228 Butler, J. 10

Cabrera, P. 359 Cabula,O.S. 104 Calvo,P. 107 Caminero, J. 359 Cases Viedma, E. 57

Chan,J.C.K. 80 Chan,T.K. 80 Chiu,E.K,W. 80 Coen,E. 355 Cole, R.P. 290 Constantopoulos,

S.H. 101 Cordero, P. 4 Cuboni,A. 331

Dahlbäck,M. 34 Das, S. 263 Dash,R.J. 263 De Backer, W. 355 DeCock,W. 355

Demoiliopoulos, D. 336 Demoiliopoulos, J. 336

Deng, S.H. 125 Donnamaria, V. 201

Eckel, H.E. 177 Eiff,M.von 341 Eirefelt,S. 34 Eklund,A. 136 Emslander, H.-P. 163 Ertl,G. 157

Falgà-Tirado, C. 353 Falk-Filipsson, A. 130 Fatsis, M. 160 Faurschou, P. 1 Ferdoutsis, M. 286

Fernández-Garrido, M. 107 Ferrari, M. 95 Flores,J. 258 Foresti,V. 21 Franzblau, C. 76 Freitag, L. 49 Frosi, A. 331 Fruhmann,G. 209 Fujimoto, H. 89 Fujimura, M. 143 Fujishima, S. 40 Fujishima, T. 64,317 Funasako, M. 89

Gabius,H.-J. 114 Garcia Avello, A. 258 Garcia Frade, L.J. 258 García-Montero, M. 107 GilSuay,V. 57 Gitt,A. 177 Giuntini,C. 201 Gonzalez, J.M. 107 Green, M. 252 Grode,G. 1

Habscheid,W. 157 Hachiya,T. 294 Hage-Hamsten, M. van 136 Hansen, G. 117 Hara,K. 217 Hara, N. 269 Haralambopoulou, A. 174 Hauck, R.W. 163 Hayasaka, M. 294 Heimer,D. 205 Hein, R. 209 Hemsén, A. 34 Hesse, M. 341

Heubner,C.C. 167 Hidaka, K. 269 Higashimoto, Y. 89 Hiraga,Y. 84 Hirasawa, M. 84 Hirose,Y. 294 Homma, Y. 248 Honda, T. 294 Honda, Y. 64,317 Hosoda,K. 64 Humbert, B. 27

Igarashi, T. 110 Iida,K. 217

Ikeda,S. 185,237,302,348 Imaizumi, K. 155 Inoue, T. 223 Inuzuka, M. 84 Ip, M.S.M. 80 Ishida,T. 223 Ishihara,H. 274 Ishizaka, A. 40 Iwamoto,Y. 89

Jeon,S.C. 43 Jindal,S.K. 263 Jones, R.L. 70 Julià-Serdà, G. 359

Kadota,J.-i. 217 Kanazawa, M. 40 Kanel,G.C. 104 Katsuyama, T. 294 Kawahara, K. 244

Kawakami,Y. 248 Kawakani, K. 217 Kawashiro, T. 40 Kayser, K. 114 Keistinen, T. 151

Kochsiek,K. 157 Kohno,S. 217 Kopernic,G. 205 Krafte-Jacobs, B. 171 Kralingen, K. van 148

Krishnareddy, N. 104 Krombach,F. 209 Kubo, K. 294 Kubo,S. 302 Kuroki,Y. 64 Kusaka,H. 248 Kusano, S. 217 Kyriakidis, V. 101

Lakshminarayan, S. 10,307 Lam,W.K. 80 Lampronti, G. 95 Lancha,C. 258 Låstbom, L. 130

Lazer, S. 205 Lee,D.H. 43 Lee,J.D. 43 Lee,J.H. 43 Leon Fábregas, M. 57 Levitas, E. 205

Lieberman, D. 205 Linke,R.P. 163 Liu,L.H. 125 LoCascio, V. 95 Loo, J. vande 341 Loza,E. 107

Lundberg, J.M. 34 Lymberopoulos, D. 174

Macián, V. 4 Mady,B.J. 232 Maehara,Y. 223 Man,G.C. 70 Manganelli, D. 201 Maniati,M.A. 101 Marco, V. 312 Marek,W. 322 Martinez Moragón, E. 57 Massaia,M. 228 Matsuda, T. 143 
Meletis,G. 286 Menéndez, R. 312 Metersky, M.L. 234 Milman, N. 1 Mitsuyama, T. 269

Miyahara, Y. 185,237,302,

348 Moldéus,P. 130 Molero,T. 359 Montero, C. 53 Morales, P. 4 Mori,M. 40 Morikawa,T. 217

Morris, T.A. 11176 Moschos, M. 336 Moskowitz, H. 234 Munakata, M. 248 Muraoka, M. 244

Murate,T. 155 Myou,S. 143

Naito,T. 185,237,348 Naitoh,T. 302 Nakai,S. 89 Nakamura, A. 244 Nakamura,K. 237

Nakamura, Y. 89 Nakanishi,F. 84 Nathrath, W.B.J. 163 Neary,J.M. 70 Nishikawa, K.-i. 274

Ogasawara, H. 248 Ohata,M. 89 Ohmichi, M. 84 Ohno,J. 155 Öhrn,M.B. 136 Ohtsuka,Y. 248

Olivieri,M. 95 Ordi-Ros,J. 353 Ozawa,K. 274

Palla,A. 201 Palumbo,R. 16 Panagou,P. 280,336 Panayiotides, J. 160 Pang, Y.Y. 125

Papiris, S.A. 101 Paraskevopoulos, A. 336 Park,S.S. 43 Patsourakis, G. 286 Pérez-Pemán, P. 353

Perez Rodriguez, E. 258 Perpiñá Tordera, M. 57 Petruzzelli, S. 201 Petsas,T. 174

Philippou, S. 49 Pombo Felipe, F. 53 Porath,A. 205 Postmus,P.E. 148 Präuer,H.W. 163

Radice,L. 331 Rodriguez de Castro, F. 359 Rodríguez-García, J.L. 107 Rolla,G. 228 Rominger,

K.L. 190 Roos,N. 341 Rubio,V. 312 Ryan,T.G. 70 Ryrfeldt,Å. 130

Saito,M. 143 Sanchis Aldás, J. 57 Schmidt, E.W. 190 Schmidt, M. 157 Schramel,F. 148

Schulten,R. 341 Schulz,C. 163 Schulz,V. 114 Schuster, A. 117 Schwaiblmair, M. 209 Scott, M. 252 Serrano, M. 107 Shigehara,K. 84 Shijubo,N. 64,84,317 Shimokata, K. 155 Shin,D.H. 43

Siafakas, N.M. 280, 286, 336 Sigurdardottir, O. 136 Sköld,C.M. 136

Snider, G.L. 76,297 Sole, A. 312 Soler,JJ. 4 Solomou, A. 174 Song,W.D. 125 Spiliopoulou, M. 174 Spiropoulos, K. 174 Starcher, B. 252 Stone, P.J. 76 Streckert, H.-J. 27 Sugimachi, K. 223

Sugio,K. 223 Suruda,T. 89 Sutedja,G. 148

Tagawa, T. 244 Takahashi,H. 64,317 Takahashi, T. 244 Tamura, K. 274 Tanaka,H. 110

Tanaka,T. 269 Tanimura, K. 248 Tasaka,S. 40 Teramoto, S. 110 Thayer,J.O. 234 Theegarten, D. 49 Todisco, T. 16 Tomita,M. 244 Toro Santos, F.J. de 53 Torres Lanzas, J. 53 Tzanakis, N. 280 Uetani,K. 89 Ulmer,W.T. 190,322

Van de Heyning, P. 355 Verbeke,C.S. 167 Verbraecken, J.A. 355 Verea-Hernando, H. 53

Vezzoli,F. 331 Vezzoli,S. 331 Vilkman,S. 151

Wahn,V. 117 Wassermann, K. 177 Weber, E. 27 Werchau,H. 27 Weyde,J. 177 Whitman, B.W. 171 Wiebel,M. 114 Willemen,M. 355 Wirtz,H. 157

Xiao,Z.L. 307

Yamamoto, S. 244 Yamasawa, F. 40 Yiatromanolakis, N. 336 Yoshida,Y. 110 Yoshioka, K. 46 Yuen,K,Y. 80

Zackrisson, C. 34 Zar,H.J. 290 Zetterström, O. 136 Zhang, A.C. 125 Zhang, S.Y. 125 Zhao,J.Y. 125 Zubrod,C. 117 Zühlsdorf,M. 341

362

Author Index 